\title{
OR-73
}

\section{Separation of Saponin Glycosides from Asparagus Racemosus by High Speed Countercurrent Chromatography}

\author{
Churanya Onlom ${ }^{1,2,{ }^{*}}$, Yi Yang ${ }^{3}$, Haji Akber Aisa ${ }^{3}$, Neti Waranuch ${ }^{2}$ and Kornkanok Ingkaninan ${ }^{1,2}$ \\ ${ }^{1}$ Bioscreening Unit, Department of Pharmaceutical Chemistry and Pharmacognosy, Faculty of Pharmaceutical Sciences \\ and Center of Excellence for Innovation in Chemistry, Naresuan University, Phitsanulok 65000, Thailand; ${ }^{2}$ Cosmetic \\ and Natural Product Research Unit, Faculty of Pharmaceutical Sciences, Naresuan University, Phitsanulok 65000, \\ Thailand; ${ }^{3}$ Xinjiang Key Laboratory of Plant Resources and Natural Products Chemistry, Xinjiang Technical Institute of \\ Physics and Chemistry, Chinese Academy of Sciences, Urumqi 830011, China; E-mail: churanyaO@gmail.com
}

High-speed countercurrent chromatography (HSCCC) was successfully applied for the first time for the separation and purification of two saponin glycosides i.e. shatavarin IX and asparagoside A from roots of Asparagus racemosus Willd. Shatavarin IX was obtained from HSCCC using two-phase solvent system, chloroform-methanol-water with the lower phase as the mobile phase. Asparagoside A was isolated using a solvent system, ethyl acetate-n-buthanol-water with the lower phase as the mobile phase followed by the separation via Sephadex LH20. From $500 \mathrm{mg}$ of crude extract, $7.3 \mathrm{mg}$ of shatavarin IX and $6.4 \mathrm{mg}$ of asparagoside A were obtained at purities of $93.9 \%$ and $88.3 \%$, respectively. The chemical structures were elucidated by ESI-MS and NMR. Shatavarin IX shows mild antifungal activity against Candida albicans.

Keywords: Asparagus racemosus, Antifungal, High speed counter current chromatography, Saponin glycosides. 